\title{
Perforated duodenal diverticulum: a report of two cases
}

\author{
W.S.L. Stebbings and James P.S. Thomson \\ Department of Surgery, Hackney Hospital, London E9 6BE, UK.
}

\begin{abstract}
Summary Perforation is a rare complication of congenital duodenal diverticula. The case histories of 2 patients are reported. Operative treatment to excise the diverticulum and close the defect in the duodenum and to drain associated sepsis is required.
\end{abstract}

\section{Introduction}

Two cases of perforated duodenal diverticulum that presented to a district general hospital within a period of 4 weeks are reported. Duodenal diverticula are not uncommon, radiological evidence suggesting an incidence of up to $5 \%$ of the population (Neil \& Thompson, 1965), whereas post-mortem data has revealed an incidence as high as $22 \%$ (Ackerman, 1943). Perforation, however, is a rare but serious complication.

\section{Case reports}

\section{Case 1}

A 67 year old man was admitted with a $12 \mathrm{~h}$ history of sudden onset of colicky upper abdominal pain radiating through into his back. He was nauseated and had vomited repeatedly. Nineteen years previously he had undergone a Billroth II gastrectomy for chronic duodenal ulcer disease. On examination he was thin, appeared unwell, and was pyrexial with a tachycardia. There was generalized abdominal tenderness which was maximal in the right flank. Bowel sounds were present. Abdominal $\mathrm{X}$-rays showed multiple small bowel fluid levels with no gross dilatation and there was no free gas in the peritoneal cavity. He was initially treated for $38 \mathrm{~h}$ with intravenous fluids and nasogastric suction with a presumptive diagnosis of small bowel obstruction due to adhesions. During this period he developed an area of erythema in the right flank. His clinical condition did not improve and it was therefore decided to proceed to laparotomy. At laparotomy a large retroperitoneal abscess around the duodenum and lower pole of the right kidney was found. The duodenum was mobilized and a perforated

Correspondence: J.P.S. Thomson M.S., F.R.C.S., St Mark's Hospital, City Road, London EC1V 2PS, UK.

Accepted: 3 April 1985 duodenal diverticulum was found. The diverticulum was excised and the duodenum closed in two layers. The abscess cavity was evacuated and drained with a large tube drain.

His postoperative recovery was complicated by the development of a duodenal fistula on the 7th postoperative day, which was of relatively low output, averaging $500 \mathrm{ml} / \mathrm{d}$. Parenteral feeding was commenced and on this treatment the fistula closed on the 18th postoperative day and a complete recovery ensued.

Case 2

An 89 year old widow presented with a $24 \mathrm{~h}$ history of sudden onset upper abdominal pain that had localized to the right side. On examination she had marked tendernesss and guarding in the epigastrium and right upper quadrant. Abdominal X-rays showed no free intraperitoneal gas. Electrocardiogram revealed complete heart block. She was initially treated conservatively with intravenous fluids and antibiotics with a provisional diagnosis of acute cholecystitis. However, ultrasound on the following day failed to confirm the presence of gallstones, and as there had been no improvement in her clinical condition it was decided to proceed to laparotomy. At laparotomy a retroperitoneal inflammatory process lateral to the duodenum was found, with associated retroperitoneal bile staining, oedema and emphysema. The duodenum was mobilized, and a perforated duodenal diverticulum found. The diverticulum was excised and the duodenum closed in two layers. The retroduodenal space was drained with a closed tube drain. She made an uneventful postoperative recovery but has remained in hospital under geriatric care.

\section{Discussion}

Duodenal diverticula are either congenital or acquired, and the latter may be classified as primary or

(C) The Fellowship of Postgraduate Medicine, 1985 
secondary types. Primary diverticula, as in the patients reported here, are always found distal to the first part of the duodenum, consist of mucosal and serosal layers only and are thought to be pulsion diverticula. Secondary diverticula consist of all layers of the wall and are thought to be due to traction as the result of peptic ulcer disease. Primary diverticula may be single or multiple $(20 \%)$ and $95 \%$ occur on the concave border of the second and third parts of the duodenum. However, they are usually asymptomatic and only require surgery for the complications.

Perforation which is the most serious complication is fortunately the rarest. One series reports only 2 cases out of 70,000 surgical admissions over 20 years (Juler et al., 1969). The exact pathogenesis of perforation is not fully understood, but it is thought that it is due to stasis and peptic activity within the sac. Pre-operative diagnosis of the condition is difficult, because there are no pathognomonic symptoms and signs. As the diverticula are retroperitoneal structures there is no free gas radiologically. It is therefore very important to recognize the significance of localized retroperitoneal air over the area of the duodenum and upper pole of the right kidney (Wolfe \& Pearl, 1972). If there is a frank abscess there may be small fluid levels in the area as well, which were noted retrospectively in the first of our cases. The first case developed a specific physical sign, namely a small area of erythema in the right

\section{References}

ACKERMAN, W. (1943). Diverticula and variations of the duodenum. Annals of Surgery, 117, 403.

DONALD, J.W. (1979). Major complications of small bowel diverticula. Annals of Surgery, 90, 183.

JULER, G.L., LIST, J.W., STEMMER, E.A. \& CONNOLLY, J.E. (1969). Perforating duodenal diverticulitis. Archives of Surgery, 99, 572. flank. This can only be interpreted as indicative of retroperitoneal collection of pus and not as pathog. nomonic of perforated duodenal diverticulum. However, this sign does not appear to have been mentioned in previous publications.

At laparotomy the diagnosis is not always im? mediately obvious, and Juler et al (1969) have founc. that bile stained phlegmon in the paraduodenal areas? retroperitoneal oedema and emphysema lateral to the duodenum, and retroperitoneal pus in the area of the्छ duodenum are useful clues.

The surgical management of choice involves mobilization of the duodenum to expose the diver $\vec{\omega}$ ticulum. Then the anatomy of the ampulla and the distal end of the bile duct and their relationship to theo neck of the sac should be defined. This can be aided by? the passage of a probe into the ampulla either via as duodenotomy or from above via a choledochotomy:The diverticulum can then be excised and the defect ino the wall closed in two layers. The suture line may bew reinforced with an omental patch, and the re- 0 troduodenum should be drained. Complications of primary resection and closure include biliary obstruc $-\overrightarrow{-}$ tion, pancreatitis, and duodenal fistula formations (Donald, 1979). The first two of these can probably be prevented by careful definition of the anatomy to prevent damage to the ampulla and the distal end of the bile duct, combined with T-tube decompression.

NEIL, S.A. \& THOMPSON, N.W. (1965). The complications of duodenal diverticula and their management. Surgery Gynecology and Obstetrics, 120, 1251.

WOLFE, R.D. \& PEARL, M.J. (1972). Acute perforation of duodenal diverticulum with roentgenographic demonstra tion of localised retroperitoneal emphysema. Radiology, 104, 301. 